\title{
Hot-Spots in Plasma-Focus Discharges as Intense Sources of Different Radiation Pulses
}

\author{
L. Jakubowski and M.J. Sadowski \\ The Andrzej Soltan Institute for Nuclear Studies \\ 05-400 Otwock-Swierk by Warsaw, Poland.
}

Received on 26 June, 2001

\begin{abstract}
The paper concerns pulsed discharges of the Plasma-Focus (PF) type, and in particular the formation of micro-regions (so-called hot spots) with very high electron concentration and relatively high electron temperature. Particular attention is paid to the correlation between X-ray pulses emitted from the hot spots and the emission of pulsed electron beams, ion beams, and the pulsed neutron fluxes.
\end{abstract}

\section{Introduction}

It is well known that a current sheath layer, which is formed by a high-current discharge within a PlasmaFocus (PF) system, undergoes first the axial acceleration, and after reaching the electrode ends it is subject to the radial collapse [1-3]. This process leads to the formation of the dense magnetized plasma column on the z-axis. In such a column there appear magnetohydrodynamic $(m=0)$ instabilities, which produce local plasma regions of small dimensions (of the order of several or dozen micrometers), but with high electron concentrations $\left(10^{20}-10^{21} \mathrm{~cm}^{-3}\right)$ and relatively high electron temperatures $(0.5-5 \mathrm{keV})$ [4]. Such plasma micro-regions, so-called "hot spots", are well visible particularly in PF discharges performed within a hydrogen (or deuterium) mixture with some heavy gases (e.g. argon). The hot spots are identified as sources of intense X-ray emission as well as pulsed electronand ion-beams [5]. If the PF discharges are carried out within pure deuterium, as a result of nuclear fusion D$D$ reactions, there are emitted also neutron pulses with the total yield, which is a measure of the PF discharge quality. The main aim of this paper was to study a correlation between the appearance of hot spots and the emission of different radiation pulses. The described experiments were carried out within the MAJA-PF device [4-5], operated at IPJ in Swierk, Poland.

\section{Experimental setup}

The MAJA-PF facility was equipped with two coaxial electrodes of the Mather type. These electrodes are 72 $\mathrm{mm}$ and $124 \mathrm{~mm}$ in diameter, respectively. The both electrodes are $300 \mathrm{~mm}$ in length. The front plate of the inner electrode has a central 10-mm-diameter hole, which is used for the observation of pulsed electron beams emitted in the upstream direction. The electrode system is supplied from a condenser bank, which is usually operated at $45 \mathrm{~kJ}$ by the initial charging up to $35 \mathrm{kV}$. At this operational voltage, the maximum discharge current amounts to about $500 \mathrm{kA}$. During the $\mathrm{PF}$ experiments described in this paper the experimental chamber of the MAJA-PF device was filled up with pure deuterium or a mixture of deuterium and (up to $20 \%)$ argon.

\section{Experimental studies}

During numerous PF discharges performed within the MAJA-PF facility, there were performed timeintegrated and time-resolved measurements of Xrays, fast electrons, and accelerated ions, and fusionproduced neutrons.

\section{III.1 Time-integrated observations of hot spots}

According to the known process, after the application of high voltage between the electrodes a distinct plasma layer (so-called current sheath) was formed upon the insulator surface, and it was accelerated in the axial direction by the interaction of quasi-radial currents and the azimuthal magnetic field. When this current sheath reached the electrode ends it was deformed, and after that it was subject to the radial collapse. At the z-axis there was formed a dense plasma column with irregular boundaries, inside which appeared microregions (hot spots) with very high concentration and 
temperature. Some examples of the registered X-ray images are shown in Fig.1.
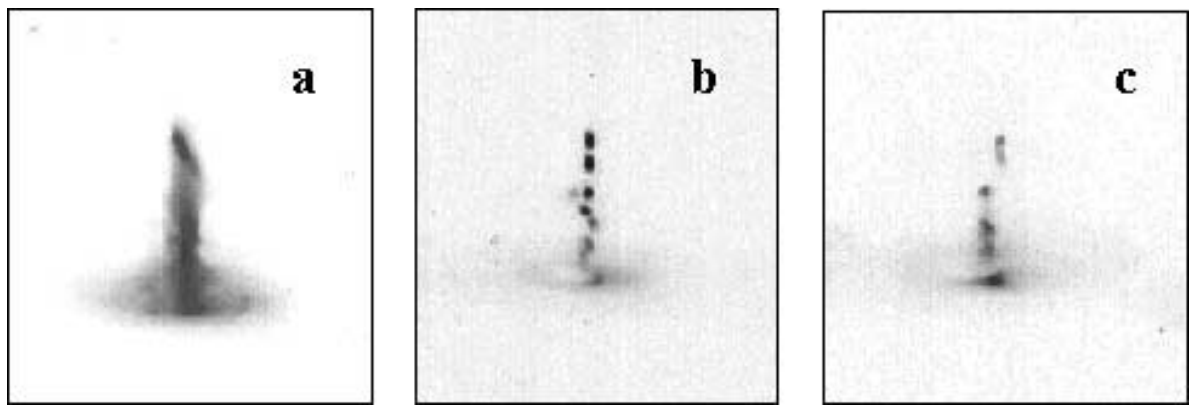

Figure 1. X-ray pinhole images from different discharges performed in the MAJA-PF facility.

Such images were registered by means of an X-ray pinhole camera equipped with a 100- $\mu$ m-diameter opening, which was covered with a $12-\mu$ m-thick Be-foil. To reduce the absorption of soft $\mathrm{X}$-rays, the camera volume (containing an X-ray film) was pumped out with an auxiliary pumping-stand (Fig.1a). It was observed that the hot spots are formed first in proximity of the electrode outlets. They have form of bright microregions surrounded by lower temperature plasma. The application of an additional absorption filter, e.g. a 5$\mathrm{cm}$-thick layer of air under atmospheric pressure, eliminates $\mathrm{X}$-rays from this lower temperature plasma. This technique enables the registration of the most intense sources, emitting X-rays of energy above $5 \mathrm{keV}$, as shown in Fig.1b. It should be noted that during a single $\mathrm{PF}$ discharge several or more (even a dozen) hot spots are formed mostly at the z-axis, although sometimes they appear also off the axis (see Fig. 1c). On the basis of the earlier studies [6], it was found that the hot spots are formed successively, starting at the electrode ends and moving as the current sheath collapses at the $\mathrm{z}$-axis. The region of the maximum compression moves along this axis (with an average velocity equal to about $3.10^{7} \mathrm{~cm} / \mathrm{s}$ ). Lifetimes of individual hot spots amount to about $10 \mathrm{~ns}$, and the hot spot population exits for about $100 \mathrm{~ns}$, counting from the moment when the collapsing current sheath reaches the z-axis. Other X-ray pinhole images, which show the formation and motion of distinct hot spots, are presented in Fig. 2.

In some cases there appear many hot spots localized closely each other within the PF pinch column, as shown in Fig. 2a. Sometimes, one can also observe the bright plasma objects moving nearby the z-axis (see Fig. 2b). The described images can be interpreted as examples of hot spots motion. Taking into account lifetimes of these hot spots and their spatial displacement, one can estimate that their velocities were of the order of $5.10^{7} \mathrm{~cm} / \mathrm{s}$. In other words, it was the phase velocity of the displacement of the maximum plasma concentration. It should be noted that spatial displacements of the hot spots have been confirmed by some inclinations of X-ray spectral lines, which were registered with crystal spectrometers. Those inclinations could result from the hot spot displacement off the z-axis and from the Doppler effect induced by the moving object. This question requires more detailed investigations.
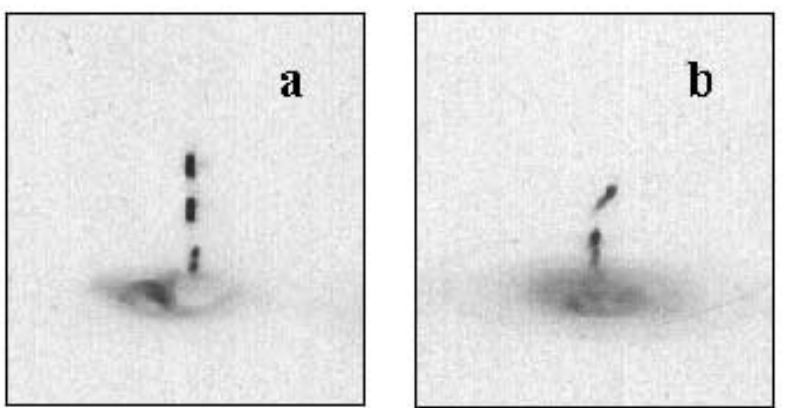

Figure 2. Examples of x-ray pinhole images showing a displacement of the distinct hot spots.

\section{III.2 Space-resolved x-ray spectra}

The high-temperature plasma produced by PF discharges, performed with a deuterium-argon filling, emits intense X-ray pulses. In order to determine values of electron temperature and concentration, one can use characteristic X-ray spectral lines corresponding to highly ionized argon ions. In the experiments within the MAJA-PF facility the use was made of a Johann-type spectrometer, which was equipped with a quartz crystal bent cylindrically. This X-ray spectrometer made possible the registration of spectral lines in the range from $3.8 \mathrm{~A}$ to $4.2 \AA$. The optical axis of the crystal was perpendicular to the $\mathrm{PF}$ pinch column. To observe $\mathrm{X}-$ ray spectral lines from individual hot spots distributed along the $\mathrm{z}$-axis, the use was made of an additional slit of $600 \mu \mathrm{m}$ in width, which was placed in front of the spectrometer. This slit ensured a satisfactory spatial resolution to register X-ray spectra from the separate hot spots. These spectra were compared with the X-ray pinhole pictures, as shown in Fig. 3. 

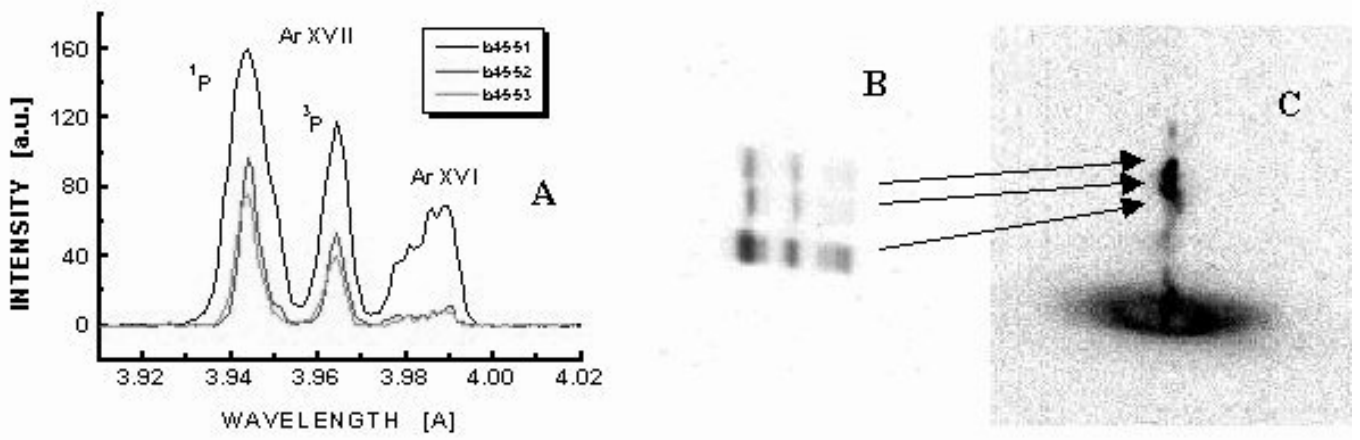

Figure 3. X-ray lines intensities (A) and corresponding X-ray spectra (B), as obtained from highly ionized argon-ions, in a comparison with the X-ray pinhole picture $(\mathrm{C})$, which shows the spatial distribution of hot spots in the investigated $\mathrm{PF}$ discharge.

Table 1: Results of the spectral measurements.

\begin{tabular}{|c|c|c|c|c|c|}
\hline $\begin{array}{c}\text { Spectrum } \\
\text { number }\end{array}$ & $\mathrm{I}_{R}$ & $\mathrm{I}_{I}$ & $\mathrm{I}_{S}$ & $\mathrm{n}_{e}\left[\mathrm{~cm}^{-3}\right]$ & $\mathrm{T}_{e}[\mathrm{eV}]$ \\
\hline \hline $45-51$ & 195.0 & 137.7 & 89.9 & $3^{*} 10^{20}$ & 255 \\
$45-52$ & 109.8 & 60.0 & 13.9 & $1.6^{*} 10^{21}$ & 790 \\
$45-53$ & 86.1 & 52.9 & 12.9 & $1.0^{*} 10^{21}$ & 650 \\
\hline
\end{tabular}

The space-resolved X-ray spectra (shown in Fig.3a,b) originated from different hot spots formed along the discharge axis. The comparison of those spectra with the corresponding X-ray pinhole picture (Fig. 3c) confirmed that they were evidently emitted from different hot spots. The described measurements showed that the most intense were helium-like argon lines, and in particular the resonance line ArXVII- ${ }^{1} P$, the intercombination line $\mathrm{ArXVII}^{3}{ }^{3} P$, and satellite lines ArXVI, as presented in Table 1.

The values of the electron concentration $n_{e}$ were estimated on the basis of the $\mathrm{I}_{R} / \mathrm{I}_{I}$ ratio [7] observed for individual hot spots. The obtained values were different, as shown in Table 1, but they changed within the range of $10^{20}-10^{21} \mathrm{~cm}^{-3}$. It should be noted that these values were calculated under assumption that electrons have the Maxwellian distribution function. Therefore, the results must be treated as approximate ones. In order to determine these parameters with better accuracy, one need data about directed electron beams and their influence on the population of different energy levels in the argon ions. The values of the electron temperature $T_{e}$ were calculated from the ratio of satellite lines ArXVI and the resonance line ArXVII- ${ }^{1} P$ for individual hot spots. The results presented in Table 1 show that hot spots (formed within the same PF discharge) can have different electron temperatures $(200-700 \mathrm{eV})$. It should be noted that such electron concentration- and temperature-values were determined for many PF discharges and numerous hot spots. On the basis of a comparative analysis of subsequent hot spots it was found [4] that the formation of the hot spots has a stochastic character. The locations as well as the electron concentration and temperature values seem to be independent of neighboring hot spots. A completely different conclusion was obtained when the $n_{e}$ and $T_{e}$ values were compared as a function of a distance from the electrode outlet, as shown in Fig. 4.
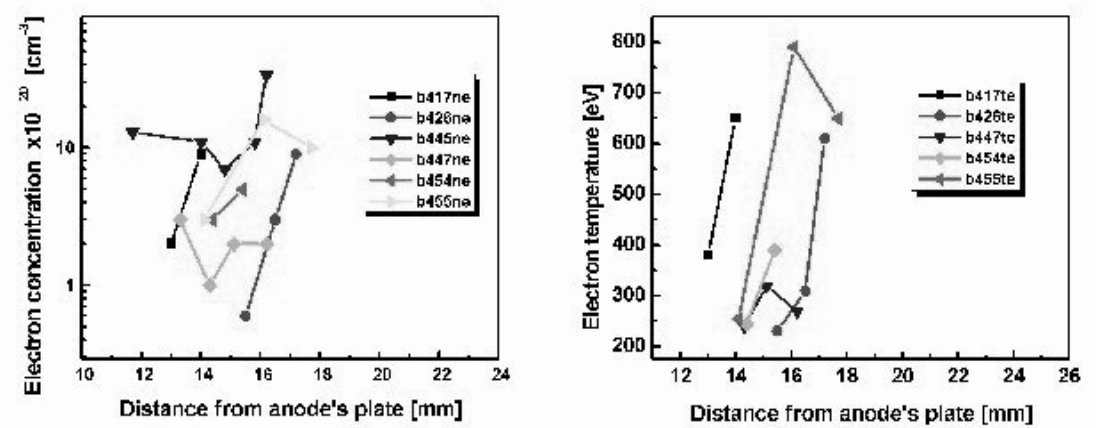

Figure 4. Electron concentration $n_{e}$ and electron temperature $T_{e}$ values, which were computed for subsequent hot spots in different PF discharges, as a function of their distances from the end plate of the inner electrode (anode). 
The presented experimental data show that the $n_{e}$ and $T_{e}$ values increase for the subsequent hot spots if they are formed in a small distance (about $1 \mathrm{~mm}$ ). One could suspect that this is due to some ionization and heating up the plasma in the close neighborhood. From physical point of view it might be possible, since (under assumption that the axial velocity of the maximum concentration in the collapsing current sheath is about $3.10^{7} \mathrm{~cm} / \mathrm{s}$ ) the formation of the next hot spot at the distance of $1 \mathrm{~mm}$ takes several nanoseconds only. This time is shorter than the lifetime of the previous hot spot. The above interpretation does not refer to all the cases because there appear different local conditions (e.g. strong electrical and magnetic fields), but this question requires more detailed investigations.

\section{III.3 Studies of pulsed electron beams}

The appearance of local strong electromagnetic fields within the PF pinch column (i.e. inside or nearby the hot spots) was confirmed by observations of intense electron beams, emitted mainly in the upstream direction (towards the anode). The earlier experimental studies [9] showed the emission of X-ray pulses from the observed hot spots is accompanied by the emission of pulsed electron beams with a relatively wide energy spectrum. These phenomena were also analyzed on the basis of a theoretical model assuming the generation of so-called "run away" (fast) electrons. The model computations were performed for the experimental conditions occurring in the MAJA-PF facility, and their results were in agreement with the observed emission of pulsed electron beams [10]. Experimental studies of the fast electron beams in the MAJA-PF device were carried out by means of Cerenkov-type detectors containing the radiators made of quartz or rutil crystals. These particular materials were applied because of their low energy threshold (equal to about $50 \mathrm{keV}$ ). It was found that the fast electron pulses can last about 7-10 ns, and the number of such pulses depends on an amount of the appearing hot spots. The emission of the electron pulses was observed during about $100 \mathrm{~ns}$. In order to investigate an energy distribution of the emitted elec- trons, the use was made of a small magnetic analyzer with a deflection equal to $180^{\circ}$. It was placed behind the axial channel drilled in the inner electrode. The detailed measurements made possible to determine energy spectra of the investigated electron beams. They extended from about $5 \mathrm{keV}$ to above $600 \mathrm{keV}$, having a distinct maximum near $50 \mathrm{keV}$. The registered energy spectra had some local peaks, which could be attributed to separate hot spots under the assumption that each hot spot emits electrons within a relatively narrow energy range [5].

Time-resolved measurements of electron pulses were performed by means of miniature scintillation detectors, which were fixed upon the detection plane of the same magnetic analyzer. It made possible to observe temporal changes of the electron emission in the selected energy ranges. A comparison of the registered electron signals confirmed that individual pulsed electron beams are emitted from different hot spots, which are formed with some time delays. Detailed investigations of energy spectra of the pulsed electron beams, emitted from different hot spots, have shown that:

1. The electron energy spectra from the PF discharges, which were carried out with pure deuterium, have extended over a relatively wide range from about $10 \mathrm{keV}$ to about $600 \mathrm{keV}$. On contrary, the spectra registered for PF shots performed with an argon admixture (up to 20\%) have been limited to a relatively narrow energy band.

2. Energy ranges of the electron beams from different hot spots have varied from shot to shot under the same experimental conditions. An example of oscillograms, which were registered for two successive PF discharges within the MAJA-PF facility, is presented in Fig. 5 .

During the first shot there were emitted electrons of energy 200-500 keV, while during the second shot (performed at the same conditions) only low energy (20-50 $\mathrm{keV}$ ) electrons were observed. It should be noted that in the both cases the time-integrated X-ray pinhole images were very similar.
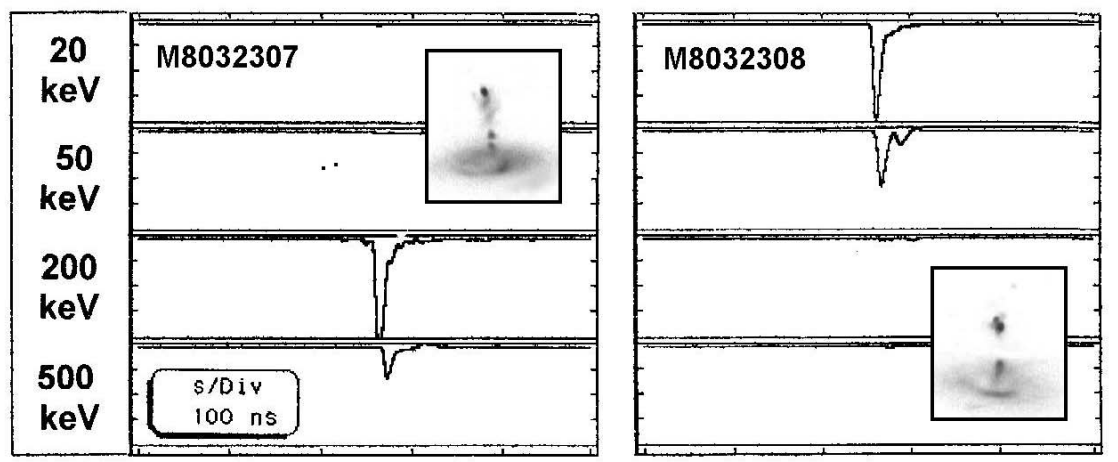

Figure 5. Comparison of electron pulses, emitted from two successive PF shots within the MAJA-PF facility and measured within different energy channels. Inserted are the corresponding X-ray pinhole pictures, which show the location of the observed hot spots. 
3. One could suspect that the pulsed electron beams emitted from the hot spots, which were formed within the PF discharges with the deuterium-argon mixture, have had different energy characteristics in dependence on the place and time of their generation. In general, the hot spots, which were formed later (at a larger distance from the anode end), have emitted the electron beams of higher energy, as shown in Fig. 6.

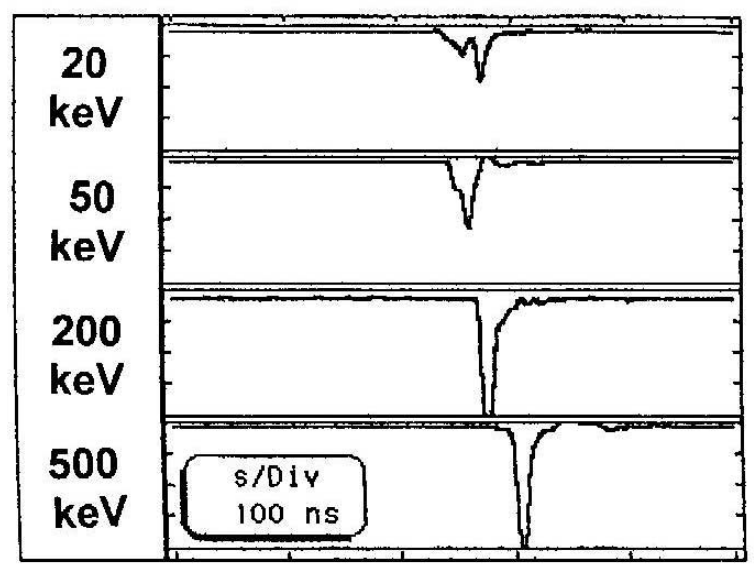

Figure 6. Increase in energy of the pulsed electron beams emitted by the hot spots, which were formed successively during the PF discharge within the MAJA-PF device.

\section{III.4 Studies of pulsed ion beams}

In order to investigate the correlation between the formation of the hot spots and the pulsed emission of ion streams, the use was made of an ion pinhole camera equipped with solid-state nuclear track detectors [11]. This camera was adjusted along the z-axis, and its inlet diaphragm was placed at a distance of $280 \mathrm{~mm}$ from the inner electrode (anode) end plate. An analysis of the irradiated and etched detectors made possible the investigation of a spatial distribution of the registered ion beams, and the determination of absolute values of the ion fluxes. Examples of the ion images and corresponding electron-induced signals, which were obtained from a single PF discharge in the MAJA-PF facility, are presented in Fig. 7.

On the basis of a comparison of the ion pinhole images (registered along the z-axis) and the corresponding electron signals (registered in the upstream direction) one could suspect the simultaneous emission of the investigated ions and fast electrons [11]. Considering the experimental results presented in Fig. 7, it seems reasonable to assume that the pulsed ion beams are emitted from different miniature ion sources, which are distributed along the PF pinch column and can be identified with successive hot spots.

From the detailed analysis of the ion pinhole pictures, which were taken for the investigated PF discharges performed within the MAJA-PF facility, it was estimated that the total ion flux amounted to about $10^{12}$ deuterons/stereo-radian. It should also be noted that recently some efforts have been undertaken to analyze the correlation of the hot spots with the emission of fast neutrons (produced by D-D fusion reactions). For this purpose a neutron scintillation detector has been placed nearby the MAJA-PF experimental chamber, and neutron-induced signals have been transmitted to a fast photo-multiplier (through an appropriate optical cable). The experimental results obtained so far have appeared to be difficult for the interpretation, because neutrons scattered from the laboratory walls and equipment disturb the registered signals. Some special measures have to be undertaken to perform the studies in question.
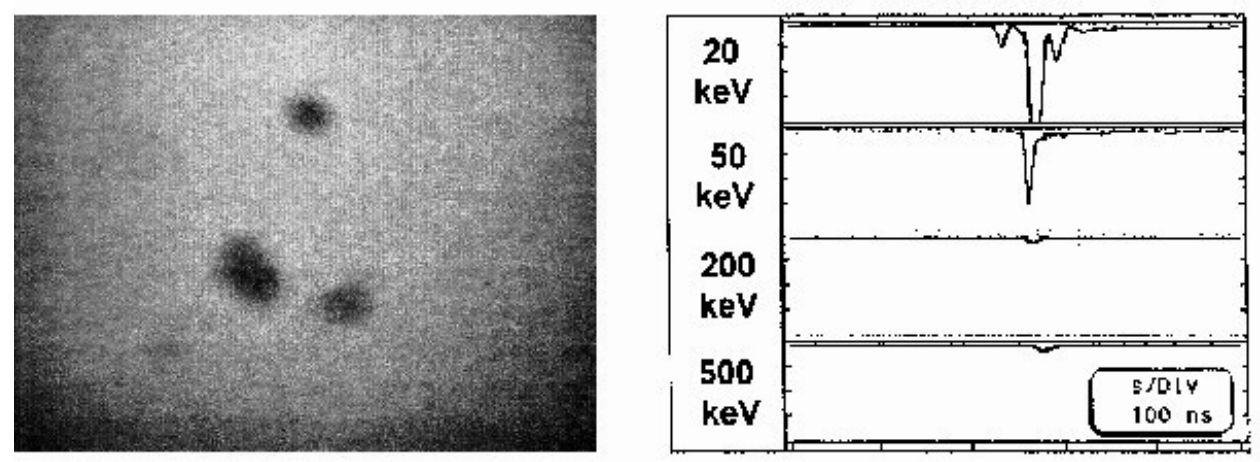

Figure 7. Ion pinhole image, which shows the tracks produced by the pulsed ion beams, and the oscillogram of the electronbeam signals, which were registered during the same PF discharge within the MAJA-PF device.

\section{Summary and conclusions}

The most important results of the experimental investigations describe in this paper can be summarized as follows:

1. The formation of several (even a dozen) of hot spots was observed in numerous PF discharges. Also 
observed was their displacement with relatively high velocities. These phenomena influenced a spectrum of the $\mathrm{X}$-ray emission from individual hot spots.

2. It was found that values of the local electron temperature and concentration for several hot spots were higher when the observed hot spots were situated close in space and time.

3. There were also measured pulsed electron beams of energies in a wide (several hundreds $\mathrm{keV}$ ) range, which were emitted from different hot spots.

4. Also measured were ion (deuteron) beams emitted along the $\mathrm{z}$-axis, and registered by means of $\mathrm{CN}$ type track detectors. Different groups of the ion tracks, as obtained after the etching of the irradiated $\mathrm{CN}$ detectors, were identified with different hot spots. An optical analysis of the registered ion tracks enabled ion fluxes (of about $10^{12}$ deuterons/stereo-radian) to be measured.

Recently, some efforts have been undertaken to investigate time correlation of fast neutron pulses, which originate from D-D fusion reactions occurring inside or nearby different hot spots. Such measurements require more detailed studies, because disturbances induced by scattered neutrons must be eliminated.

\section{References}

[1] N.V. Filippov, T.I. Filippova, and V.P. Vinogradov, Nucl. Fusion Suppl. 2, 577 (1962).

[2] J.W. Mather, Phys. Fluids Suppl. 7, 5 (1964).

[3] A. Bernard, H. Bruzzone, P. Choi, H. Chuaqui, et al., J. Moscow Phys. Soc. 8, 93 (1998).

[4] L. Jakubowski, M. Sadowski, and E. O. Baronova, Czech. J. Phys. 50(S3), 174 (2000).

[5] L. Jakubowski, M. Sadowski, E. O. Baronova, and V.V. Vikhrev, Proc. Intern. Conf. on High-Power Particle Beams (Haifa, 1998), Pt. 2, p. 615.

[6] L. Jakubowski, and M. Sadowski, Proc. 22nd EPS Conf. CF\&PP (Bournemouth, 1995), Pt. 2, p.181.

[7] A.V. Vinogradov, et al., Kvantovaya electronika (in Russian) 2, 1165 (1975).

[8] E.V. Aglickiy, et al., Kvantovaya electronika (in Russian) 1, 579 (1974)

[9] L. Jakubowski, and M. Sadowski, Proc. 1996 Intern. Conf. on Plasma Phys. (Nagoya, 1996), Pt. 2, p.1326.

[10] L. Jakubowski, M. Sadowski, E. O. Baronova, and V.V. Vikhrev, Proc. 4th Intern. Conf. on Dense Z-Pinches (Vancouver, 1997), p.443.

[11] L. Jakubowski, M. Sadowski, and J. Zebrowski., Proc. 18th Fusion Energy Conference (Sorrento, 2000); Nuclear Fusion (2001) - in print. 